\title{
HRTEM Image Simulations of Structural Defects in Gate Oxides
}

\author{
S. Taylor ${ }^{1,3}$, J. Mardinly ${ }^{2}$, M.A. O'Keefe ${ }^{3}$, and R. Gronsky ${ }^{1,3}$ \\ ${ }^{1}$ Department of Materials Science and Engineering, University of California, Berkeley, CA 94720 \\ ${ }^{2}$ Intel Corporation, Materials Technology Department, Santa Clara, CA 95052 \\ ${ }^{3}$ National Center for Electron Microscopy, Lawrence Berkeley National Laboratory, Berkeley, CA 94720
}

DISCLAIMER

This document was prepared as an account of work sponsored by the United States Government. While this document is believed to contain correct information, neither the United States Government nor any agency thereof, nor The Regents of the University of California, nor any of their employees, makes any warranty, express or implied, or assumes any legal responsibility for the accuracy, completeness, or usefulness of any information, apparatus, product, or process disclosed, or represents that its use would not infringe privately owned rights. Reference herein to any specific commercial product, process, or service by its trade name, trademark, manufacturer, or otherwise, does not necessarily constitute or imply its endorsement, recommendation, or favoring by the United States Government or any agency thereof, or The Regents of the University of California. The views and opinions of authors expressed herein do not necessarily state or reflect those of the United States Government or any agency thereof, or The Regents of the University of California.

Ernest Orlando Lawrence Berkeley National Laboratory is an equal opportunity employer.

Ernest Orlando Lawrence Berkeley National Laboratory - LBNL-47221 


\title{
HRTEM Image Simulations of Structural Defects in Gate Oxides
}

\author{
S. Taylor ${ }^{1,3}$, J. Mardinly ${ }^{2}$, M.A. O'Keefe ${ }^{3}$, and R. Gronsky ${ }^{1,3}$ \\ ${ }^{1}$ Department of Materials Science and Engineering, University of California, Berkeley, CA 94720 \\ ${ }^{2}$ Intel Corporation, Materials Technology Department, Santa Clara, CA 95052 \\ ${ }^{3}$ National Center for Electron Microscopy, Lawrence Berkeley National Laboratory, Berkeley, CA 94720
}

High-resolution transmission electron microscopy (HRTEM) is used extensively in the semiconductor industry for device characterization, and has become one of the highly favored techniques for characterizing the latest generation of ultra-thin gate oxides in MOSFET devices. However, relatively little is understood (either quantitatively or experimentally) about the limitations of HRTEM in detecting structural defects in gate oxides that could affect device performance. To investigate model defects experimentally, it would be necessary to construct "perfect" gate oxides, introduce defects with size and morphology known perfectly a priori, successfully make thin specimens that capture the defects, and then perform imaging experiments in the HRTEM. Since that task is virtually impossible, we have performed HRTEM image simulations to assess the visibility of various structural defects in gate oxides. The gate oxide was modeled as an amorphous silicon oxide $16.3 \AA$-thick, sandwiched between a gate and substrate. The substrate was (100) silicon viewed along the [110] direction. Typical gate electrodes of real devices are fabricated of polysilicon, but since multi-slice programs cannot easily accommodate a polycrystalline material, single crystal silicon identical to the (100) silicon of the substrate was used for the gate electrode. Crystalline defects were embedded in the gate oxide (Fig. 1) and simulated images were calculated with varying defect morphology, composition, size and orientation. We considered both hemispherical and pyramidal asperities lying on the substrate surface, as well as "bridging" defects that connect the substrate to the gate electrode. Image calculations of the sandwich structure were performed to simulate cross-sections with $\mathrm{Si}[110]$ parallel to the electron beam direction using the multislice approximation for a $200 \mathrm{kV}$ microscope with $\mathrm{C}_{\mathrm{s}}=0.5 \mathrm{~mm}^{1}$. A layer of amorphous silicon was added to the top and bottom of the specimen to account for amorphization that is known to occur during typical specimen preparation. The total specimen thickness in the beam direction was varied from $92 \AA$ to upwards of $300 \AA$ in order to understand the effect of specimen thickness on defect visibility within the experimentally-allowable thickness range.

We performed simulations with crystalline silicon defects embedded in the gate oxides. These defects were of various sizes having cylindrical, hemispherical, and pyramidal morphologies, and oriented with different low-index directions parallel to the beam direction. The simulations suggest that for a $200 \mathrm{kV}$ microscope with $C_{s}=0.5 \mathrm{~mm}$, silicon [110] is the most visible defect orientation, regardless of defect size or morphology. Silicon [111] and [100] defects were observable for very small specimen thicknesses $(<100 \AA)$ and large defect sizes $(>40 \AA)$, but were not visible for realistic specimen and defect sizes. Fig. 2 shows the effect of defect size on visibility for [110]-oriented cylindrical silicon bridging defects. The $20 \AA$ cylindrical defect is faintly detectable, whereas the $10 \AA$ defect is not. The importance of crystal orientation on defect visibility is illustrated in Fig. 3 for hemispherical surface defects with a $16 \AA$-radius. It can be seen that the [110] defect is slightly visible, the [111] defect is less visible, and the [100] defect is invisible. For specimens significantly thicker than $123 \AA$, which is typical of routine experimental work, the defects become invisible regardless of orientation. Defect visibility is consistently more pronounced at Scherzer defocus than at minimum contrast. Reducing the microscope's coefficient of spherical aberration to zero enhances the visibility of [111]-oriented silicon defects (Fig. 4), but does not noticeably improve the visibility of [100] or [110] silicon defects. Images were also simulated incorporating defects with different chemistry (such as $\mathrm{Cu}$ and voids). Hemispherical copper [111] surface defects with $10 \AA$-radius were not visible in the gate oxide even at an unrealistically low specimen thickness of $69 \AA$.

Results from this work reveal some fundamental limitations of the utility of HRTEM for characterizing defects in gate oxides, even with the new generation of $\mathrm{C}_{\mathrm{s}}$ corrected microscopes. These also results point out the importance of specimen preparation on the results that can be obtained.

\section{REFERENCES}

1. J.M. Cowley and A.F. Moodie, Acta Cryst. 10 (1957) 609.

2. Work funded by Intel Corporation. Access to computing facilities at the National Center for Electron Microscopy is gratefully acknowledged. The NCEM is supported by the Director, Office of Science, Office of Basic Energy Sciences, Material Sciences Division, of the U.S. Department of Energy, under contract No. DE-AC03-76SF00098. 


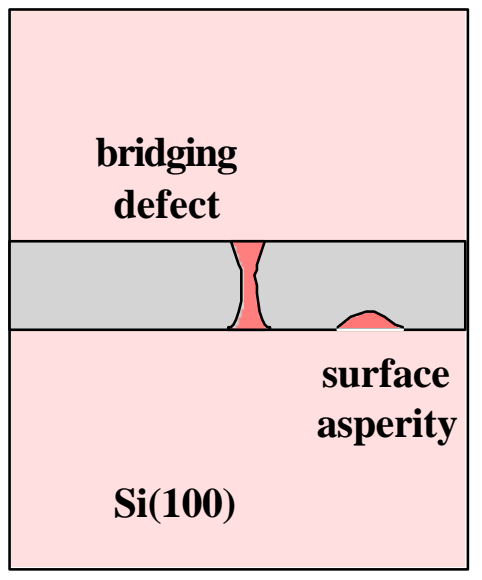

Fig.1

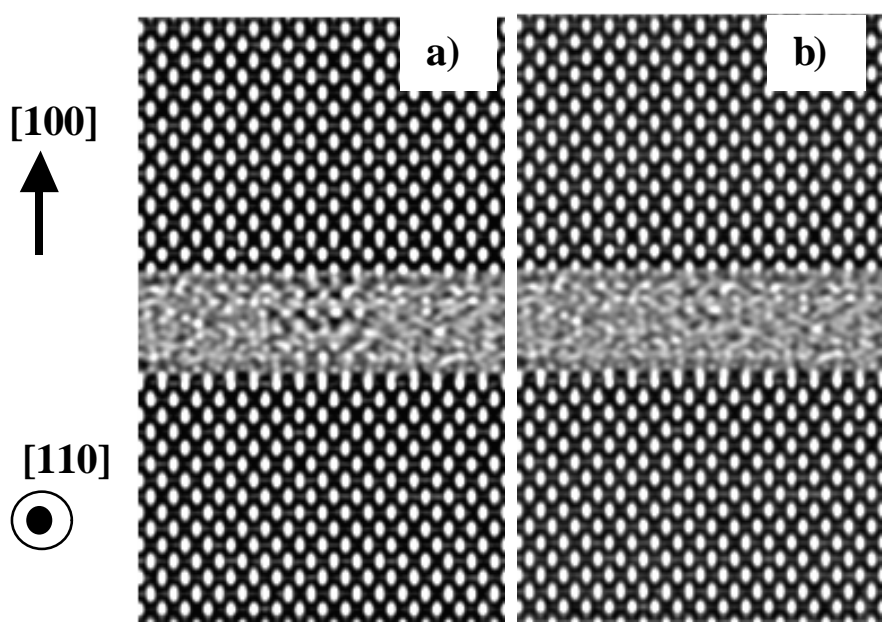

Fig.2

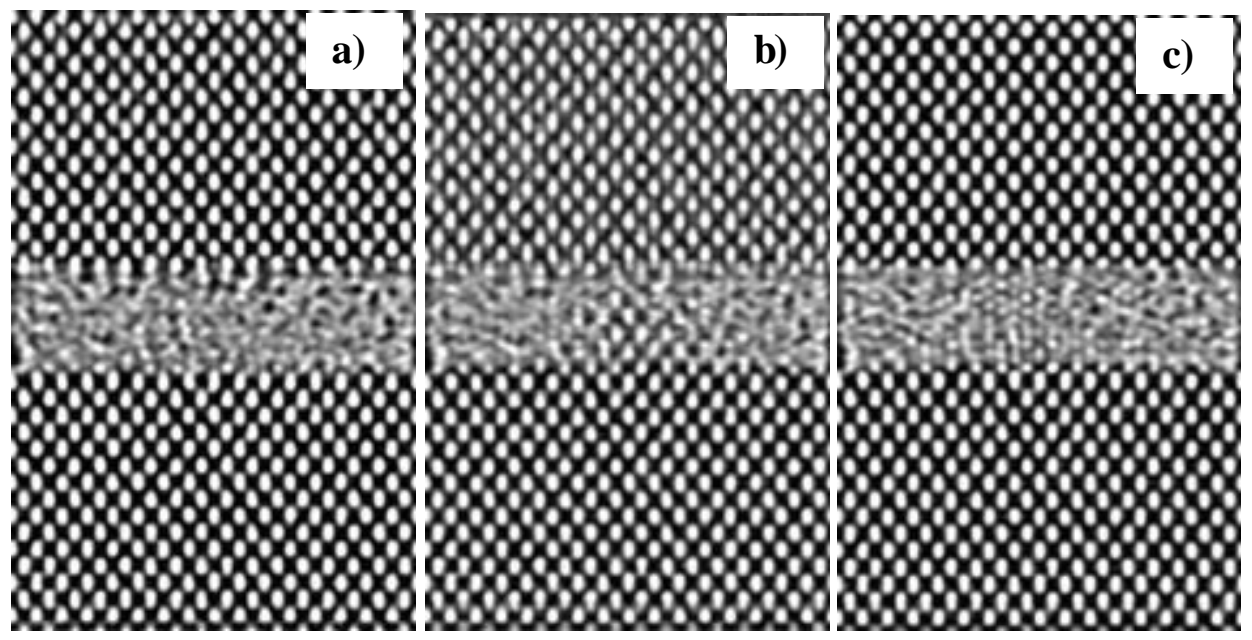

Fig.3
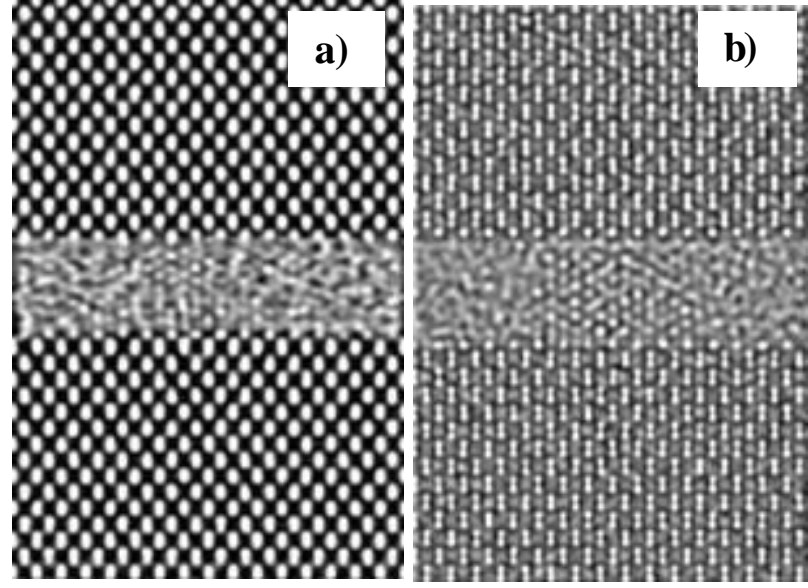

Fig.4
FIG 1 Schematic of model structure showing different types of defects.

FIG 2 Simulated images of cylindrical Si[110] defect with (a) $20 \AA$ and (b) $10 \AA$ radius (specimen thickness $=138 \AA$ ).

FIG 3 Simulated images of(a) $\mathrm{Si}$ [100], (b) Si[110], and (c) Si[111] hemispherical surface defect (radius $=16 \AA$; specimen thickness $=123 \AA$ ).

FIG 4 Simulated images of Si[111] hemispherical surface defect for (a) $\mathrm{C}_{\mathrm{s}}=0.5 \mathrm{~mm}$ and (b) $\mathrm{Cs}=0.0 \mathrm{~mm}$ (specimen thickness $=131 \AA$ ). 